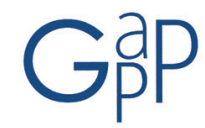

GESTIÓN Y ANÁLISIS DE POLÍTICAS PÚBLICAS, Nueva Época, nº 12 julio-diciembre 2014 ISSN: 1989-8991

DOI: http://dx.doi.org/10.24965/gapp.voi12.10209

\title{
Gobernanza y redes de políticas públicas: el caso de la política habitacional chilena
}

\author{
Olaya Grau \\ Universidad Autónoma de Chile \\ olayagrau@gmail.com
}

\section{Resumen}

En los últimos años las sociedades occidentales han experimentado profundos cambios con implicancias de distinta índole. El contexto actual se caracteriza por el dinamismo, complejidad y diversidad que solo admite una aproximación plural desde las distintas esferas de la sociedad. Ante este escenario, los gobiernos tradicionales, jerárquicos y centralizados no son suficientes ni tampoco idóneos para hacer frente a los nuevos problemas y desafíos contemporáneos. De ahí la importancia de contemplar las ideas y fundamentos que sustentan un enfoque de gobernanza democrática, el cual tiene la intención de atender las nuevas demandas y políticas públicas que las transformaciones sociales actuales requieren. Frente a lo anterior, se despliega el análisis de este trabajo que aborda el caso de la política habitacional chilena, especificamente el Programa Fondo Solidario de Vivienda (FSV). Desde este marco, el principal propósito de la investigación que sustenta este artículo, fue analizar el tipo de coordinación en red que se desarrolla en la fase de implementación de los proyectos habitacionales correspondientes al programa FSV en la Región Metropolitana (Chile) durante los años 2006 - 2010, desde un enfoque de gobernanza democrática y redes de políticas públicas.

Palabras Clave

Gobernanza; Redes; Políticas Públicas; Política Habitacional Chilena

\section{Governance and public policy networks: the case of Chilean housing policy}

\section{Abstract}

In the last years, western societies have experienced deep changes with different implications. The current context is characterized by a dynamism, complexity and diversity that must be approached from the different spheres of society. In this scenario, traditional, hierarchical and centralized governments are not enough nor suitable to face new problems and contemporary challenges. Here lies the importance of contemplating the ideas and foundations of the democratic governance approach, which intends to address the new demands and public policies that current social transformations require. The analysis will be developed, focusing on the case study of the Chilean housing policy, specifically the Fondo Solidario de Vivienda Program (FSV). The main purpose of our research was to analyze the type of network coordination that is developed during the implementation phase of FSV housing projects in the Metropolitan Region (Chile) during the years 2006 - 2010, from a democratic governance and public policy networks approach.

\section{Keywords}

Governance; Networks; Public Policy; Chilean Housing Policy 


\section{INTRODUCCIÓN}

Desde la década de los noventa, los gobiernos chilenos han puesto especial énfasis en restablecer ciertas condiciones mínimas tendientes a avanzar hacia un crecimiento con equidad (Arellano, 2012). En este sentido, durante esta década la política social jugó un importante papel en la construcción de una política de crecimiento con equidad, las que más tarde fueron denominadas políticas de protección social (Larrañaga, 2010) impulsada por los gobiernos de la concertación por la democracia (1990 - 2010)1.

Durante esta década las acciones implementadas estuvieron fuertemente orientadas a mejorar las condiciones de vida de los habitantes más pobres, a invertir en capital humano y fortalecer la base productiva (Pizarro, Raczynski, \& Vial, 1995; Arellano, 2012). Desde el año 2000 en adelante se pone el énfasis en la construcción de un nuevo sistema de protección social que se encamina hacia un enfoque más universal y basado en derechos (Abramovich, 2006).

Este artículo se enmarca en esta época de reforma, en la cual se desarrolla la nueva política habitacional chilena, política llevada a cabo por el Ministerio de Vivienda y Urbanismo (MINVU) desde el año 2006 y que tuvo como objetivo el reducir el déficit habitacional de los sectores más pobres del país, apuntando a una mejora en la calidad de las viviendas y a disminuir los índices de segregación social de los barrios, implementando mecanismos para la integración social y la participación. Esta política estuvo caracterizada por incorporar herramientas propias que responden a su vez a los enfoques de gobernanza y red de políticas públicas o policy networks, conceptos utilizados en el ámbito de gobierno para explicar la necesidad de coordinación entre los diferentes actores de la sociedad. En este sentido, la gobernanza se concretaría en redes de políticas públicas.

Bajo estos enfoques teóricos, uno de los objetivos de esta investigación se centró en conocer la existencia (o no) de una lógica de coordinación descentralizada que reconoce la autonomía de los múltiples actores de la sociedad civil que concurren en el proceso de política, en este caso en concreto, el programa Fondo Solidario de Vivienda - en adelante FSV - programa habitacional del Ministerio de Vivienda y Urbanismo, destinado a otorgar un subsidio a familias organizadas en grupos de al menos 10 integrantes, para la compra o construcción de sus viviendas.

Para esto se analizó el funcionamiento del programa FSV, en función de 6 proyectos habitacionales escogidos según el tipo de Entidades Gestoras Inmobiliaria Social (EGIS), sociedades autorizadas que operan en los programas de subsidio habitacional, entregando asistencia técnica y social en los proyectos, velando por el correcto cumplimiento del proceso y trabajando en conjunto con los potenciales beneficiarios. Pueden ser de distinta naturaleza jurídica (pública, privada y ONG/fundación).

\section{ASPECTOS METODOLÓGICOS}

Para definir los casos a abordar, en un primer momento se accedió a los registros sobre subsidios asignados por el Ministerio de Vivienda y Urbanismo (MINVU) en relación a la cartera de proyectos habitacionales postulados y seleccionados durante el año 2006, 2007, 2008 y 2009. La elección de este periodo de estudio, responde al inicio del programa FSV del año 2006, donde se establecieron las nuevas directrices de la política habitacional.

Una vez revisada la base de datos antes mencionada, se seleccionaron 3 EGIS de distinta naturaleza jurídica: una de origen público correspondiente a un municipio de la ciudad de Santiago, otra del sector privado (ámbito empresarial) y una tercera, fundación sin fines de lucro, correspondiente al tercer sector. De cada una de las entidades colaboradoras (EGIS), se seleccionaron dos proyectos habitacionales ya concluidos, donde se realizaron entrevistas en profundidad a los dirigentes, pobladores y coordinadores sociales de cada EGIS.

La elección de cada EGIS fue determinada según la cantidad de proyectos habitacionales desarrollados en la Región Metropolitana y la disposición a participar en el estudio. Para seleccionar los proyectos habitacionales se utilizó la estrategia de tipo "bola de nieve" (Coleman, 1959) de tal forma que los mismos informantes claves, en este caso, los representantes de las EGIS seleccionadas, sugirieran los proyectos que consideraban pertinentes de abordar, tomando en cuenta dos criterios claves: Subsidio asignado entre los años 2006 y 2009, y; finalización en forma exitosa del proyecto habitacional.

1 La concertación de partidos por la democracia fue constituida el 2 de enero de 1988 como coalición opositora al régimen militar establecido en Chile el año 1973. Fue compuesta por 16 partidos entre los que se pueden mencionar demócratas cristianos, radicales, socialistas, socialdemócratas, liberales, sectores de la izquierda cristiana, humanistas y el partido por la democracia. Fue la coalición de gobierno desde el año 1990 con la elección de Patricio Aylwin hasta el año 2010 con término del primer gobierno de Michelle Bachelet. 
Por tanto, el tipo de muestreo fue intencionado y queda descrito principalmente en los criterios antes explicitados: Tipo de EGIS y proyectos terminados con éxito. Este tipo de muestreo permitió obtener datos significativos para poder ir respondiendo de manera productiva a los objetivos planteados.

Se utilizó el enfoque cualitativo para acercarse a la realidad social desde los discursos producidos por los sujetos, en este caso, los distintos actores que participan en el programa FSV, poniendo especial énfasis en los beneficiarios. El optar por métodos cualitativos nos permitió permanecer próximos al mundo empírico contemplado en esta investigación. Justamente, al poner a lo cualitativo, se intentó asegurar un estrecho ajuste entre los datos y lo que la gente realmente dice y hace (Taylor \& Bogdan, 2002).

Una de las principales técnicas de recolección de información que se utilizó en esta investigación, fue la entrevista. Esta entrevista fue en profundidad de tipo semi-estructurada, por lo que se contó con un guión temático, el cual facilitó a la entrevistadora a dirigir a los entrevistados/as al campo de interés.

El análisis de la información se desarrolló en 3 niveles: Por caso, entre los casos y entre las EGIS seleccionadas. Para ello, se construyeron mapas de actores para cada uno de los proyectos habitacionales lo que permitió dar cuenta del tipo de relación, interacción e interdependencia entre los actores participantes en cada uno de los casos.

\section{MARCO TEÓRICO}

\subsection{Enfoque de gobernanza y redes de políticas públicas}

Los procesos de globalización, las crisis financieras de los Estados, la apertura y protagonismo de los mercados, han ido marcando el paso de la necesidad imperante de nuevas formas de gobernar. Podemos observar como las capacidades políticas y administrativas del sistema de gobierno han cruzado el umbral de rendimiento decreciente o están muy cerca de los límites. Por esta razón, los gobiernos han intentado reducir la necesidad de gobernar por medio de diferentes medidas como la desregulación o privatización de servicios (Kooiman, 1993). En concordancia con lo anterior, Aguilar (2006: 196) señala que "la gobernación en solitario del gobierno ha perdido sentido y eficacia. Asistimos al tránsito de un centro protagónico a un sistema de gobernación con las tres esferas que componen una nación: Estado, mercado y sociedad civil”.

Frente a esto emerge la necesidad de una alternativa que, respetando la autonomía sistémica, sea capaz de orientar su funcionamiento a la realización paralela de expectativas (Mascareño, 2010).

El concepto de la nueva gobernanza señalado por Aguilar (2006: 99) consiste en "el proceso social de decidir los objetivos de la convivencia y las formas de coordinarse para realizarlos se lleva a cabo en modo de interdependencia-asociación-coproducción/corresponsabilidad entre el gobierno y las organizaciones privadas y sociales". Aquello que trata de la decisión de los objetivos de la convivencia es precisamente lo que nortea las políticas públicas, el modo en que se abordan problemas públicos. Y como toda política pública arrastra objetivos de la convivencia, arrastraría también un referencial normativo (Jobert, 2004) que corresponde a un deber ser que guía la política.

Ahora bien, en forma sustancial y concreta, Aguilar (2006:90) entiende por gobernación/gobernanza como "el proceso mediante el cual los actores de una sociedad deciden sus objetivos de convivencia -fundamentales y coyunturales- y las formas de coordinarse para realizarlos: su sentido de dirección y su capacidad de dirección”. En este sentido, una de las formas en que se concretaría el enfoque de gobernanza democrática, sería el enfoque de redes de políticas públicas que más adelante se pondrá en discusión a partir del caso del programa de FSV de la política habitacional chilena.

\subsection{Redes de políticas públicas}

Kooiman (1993) señala que actualmente cualquier gobierno que pretenda ser eficaz debe asumir las redes y gestionar en red, impulsando un papel promotor, impulsor y cooperador. En este sentido, la sociedad de la globalización es una sociedad que opera en redes, y las redes se comunican constantemente y trabajan horizontalmente, de ahí que los modelos tradicionales de gobierno jerárquico, opaco y cerrado normalmente, ya no sean los pertinentes para gobernar (Kooiman, 1993). Lo anterior significa pensar la administración pública con públicos, con ciudadanos electores y deliberantes de las acciones que se desarrollan en este ámbito.

El concepto de red pretende ser un mecanismo para superar disfuncionalidades y reconocer la existencia de numerosos actores que inciden en las políticas públicas en un momento en el que las sociedades modernas se caracterizan por su diversidad plural y su complejidad (Cerrillo, 2005:23) 
En tanto Arenilla y Sánchez (2011:74) a partir de las definiciones de Rhodes (1997), Benson (Smith 1993:58), Börzel (1997:2) y Klinj (2005:234), entenderán por red de gobernanza o red de políticas públicas al "conjunto de actores que se relacionan entre sí de manera más o menos estable en el tiempo con la finalidad de influir, mediante el intercambio de recursos, en la definición o solución de una política pública". Klijn (1998:5) agrega que las redes de políticas forman el contexto en el que tiene lugar el proceso político, representando un intento dentro de la ciencia política para analizar la relación entre el contexto y el proceso de hechura de políticas.

El concepto de redes de políticas se podría explicar desde las relaciones estructurales, las interdependencias, la dinámica y procesos de elaboración de las políticas. Los actores de las redes interactúan pero a la vez son interdependientes y coordinan sus acciones por medio de recursos e intereses.

A partir de lo que expone Cerrillo (2005:25) se considera que las redes, en el ámbito de creación y aplicación de políticas, suponen interrelaciones, cooperación y generación de acuerdos para el logro de intereses generales, mediante el trabajo conjunto de todos los actores involucrados. "Las redes surgen por la existencia de una interdependencia entre los recursos dispersos de diferentes actores, lo que implica necesariamente el reconocimiento de interacciones entre ellos" (Cerrillo, 2005:25).

En términos aún más concretos, Arenilla y Sánchez (2011:32) sostienen que las redes son utilizadas por el poder público, para lograr mayor legitimidad y eficacia de la acción pública, y por los grupos sociales y ciudadanos para satisfacer los intereses de los actores participantes.

Mascareño (2010:116), a partir de lo que plantea Mayntz (1992), señala que el concepto de redes de políticas públicas, supone en principio la definición de reglas para la realización de compromisos entre agentes públicos y privados, que permitan una distribución de costos y beneficios frente a una decisión común o una solución de problemas; reglas que en cada caso exigen de los participantes limitar de manera voluntaria su libertad de acción; esto puede conducir a un modelo de identidades organizativas, competencias y esferas de interés mutuamente aceptadas.

Desde lo expuesto por Mascareño (2010:115) se puede entender a las redes de políticas como "el acoplamiento de la verticalidad de la democracia representativa y la horizontalidad de las múltiples formas de gobernanza de actores privados que se sitúan fuera del dominio de relaciones de la democracia representativa".

Este acoplamiento se lograría -desde lo propuesto por Koppenjan, Kars y van der Voort (2009) -a partir del desarrollo de un marco de condiciones (frameworksetting) que permita sistematizar la relación entre participantes y los límites del procedimiento en este actuar conjunto. Al hacerlo, deben enfrentarse a tres tipos de problemas: la complejidad, que se soluciona a través del diálogo; la interdependencia, que se supera mediante la adopción de un marco de condiciones como un acoplamiento suelto o loose cupling (Weick, 1976) que permita responder a posibles desviaciones; y la dinámica de todo policy issue, que se soluciona a través del aprendizaje derivado de la implementación de la política (en Mascareño, 2010: 115).

En cuanto al estudio y análisis de las redes de políticas públicas, Arenilla y Sánchez (2011) por su parte, distingue tres perspectivas centrales: (1) la que considera a las redes como una forma novedosa de intermediación de intereses, y la aplica a todos los tipos de relaciones entre actores públicos y privados; (2) la que de una manera más ambiciosa concibe a las redes como una nueva forma de gestión de gobierno denominada gobernanza, que afecta a una forma específica de interacción pública-privada en las políticas públicas, basada en la coordinación no jerárquica (Börzel, 1997:5) y (3) la que la considera una metáfora como una mera herramienta de análisis de la realidad (Dowding, 2000).

El autor opta por la segunda perspectiva para desarrollar y estudiar la red de gobernanza o políticas públicas que coincide a su vez con el enfoque que le otorgamos en este trabajo a las redes de políticas públicas.

\subsection{Características de las redes de políticas públicas}

Para que exista una red de políticas públicas se deben reconocer ciertas condiciones, que desde Arenilla y Sánchez (2011) se concretarían en 5 puntos fundamentales: en primer lugar, reconocer un marco institucional idóneo para la consolidación de redes de políticas públicas; en segundo lugar se debe distinguir cuál es la naturaleza y los tipos de interacciones de las redes; en tercero, conocer qué grado de institucionalización presentan; en cuarto lugar, en qué medida la estructura de la red condiciona los resultados de los programas/políticas; y por último, qué incidencia ha tenido el proceso en la mejora del capital social de los actores. 
El mismo Arenilla y Sánchez (2011:34) argumentan, que el modelo de redes tiene una serie de características que facilitan su estudio. La primera tiene que ver con la interdependencia de los actores, de tal modo que ningún actor puede por sí sólo alcanzar sus objetivos ni puede influir de manera decisiva sobre las políticas públicas. La segunda, se refiere a los patrones de relaciones que se establecen entre los actores, que son de interdependencia y se mantienen en el tiempo. En ese sentido las formas de relaciones son fundamentales puesto que podrían ser de subordinación, o por el contrario, deliberativas que podría responder o no a una gobernanza democrática. Por lo tanto, no cualquier patrón de relación puede ser consonante con una red que opere desde el enfoque de gobernanza. La tercera es la diversidad e importancia del número de actores que representan a grupos e intereses heterogéneos de la sociedad. La cuarta es la existencia de objetivo común a pesar de la diversidad de metas particulares. Un excesivo número de actores o cierta imprecisión en los criterios que guían las interacciones de sus miembros obstaculizan la toma de decisiones. Por último, la quinta es que uno de los actores es una organización pública que suele ser la responsable de la política en torno a la que se articula la red.

Los aspectos recién señalados serán los que más adelante se observarán en el caso concreto del programa Fondo Solidario de Vivienda de la Política habitacional chilena.

\subsection{Fundamentos de la Política Habitacional Chilena}

Desde el año 2000 con el gobierno del ex presidente Lagos se plantean los principales cambios de enfoque de la política habitacional en Chile, concretándose definitivamente bajo la denominada "Nueva política habitacional chilena", establecida a principios del 2006 que se concreta en el Decreto 174 y que se distingue básicamente por tres elementos o rasgos diferenciadores, piezas claves a la hora de argumentar por qué la política habitacional, específicamente el programa del FSV, a tratar en el presente artículo-, se podría observar desde el enfoque de redes de políticas públicas.

En primer lugar, la política por medio del programa FSV, intenta dar protagonismo a las familias beneficiarias, integrándolas como parte fundamental del proceso de ejecución del proyecto habitacional. La solución habitacional para las familias de más escasos recursos del país, se constituiría dentro de la perspectiva de redes de políticas como el gran objetivo común que comparten los actores que conforman la red.

En segundo lugar, la incorporación de las EGIS como entidades intermediarias y responsables de la asistencia técnica y social de los proyectos habitacionales. Esto sin duda, es uno de los cambios más relevantes y diferenciadores con las políticas anteriores. Por tanto, diríamos que existe interdependencia entre los actores para lograr el objetivo común.

En tercer lugar y en relación con los demás elementos antes expuestos, se distingue en la política la relación de las EGIS con el Ministerio de Vivienda, los integrantes del comité, así como también las relaciones e interacciones que se propician desde las directivas de los comités de vivienda con las propias familias beneficiarias y EGIS.

En este sentido, en cada red que conforma un proyecto habitacional se observarían patrones de relaciones e interacciones determinadas entre los actores participantes.

A partir de estos elementos diferenciadores, se despliega el análisis de este trabajo que aborda este caso en particular, donde la relevancia estaría puesta en el contexto y participación de los diferentes actores (públicos y privados) en la política habitacional.

\subsection{Principales Actores que participan del proceso del Fondo Solidario de Vivienda}

La política habitacional, específicamente el programa FSV, Modalidad Construcción en Nuevos Terrenos (CNT), contempla diferentes actores para la implementación del programa y ejecución de los proyectos habitacionales. Estos actores pueden ser gubernamentales o no gubernamentales (privados u organismos sin fines de lucro), lo que da cuenta de una diversidad de actores que se hacen parte en el desarrollo del proceso.

Es importante resaltar, que si bien se incorporan nuevos actores (EGIS) al proceso de ejecución de los proyectos habitacionales, los demás actores participaban de forma distinta o respondían a otras funciones. Lo interesante es destacar como se modifican las interacciones entre ellos y en este sentido cómo adquieren nuevos roles en esta fase de implementación.

Los principales actores públicos o gubernamentales señalados en el Decreto 174 (Ministerio de Vivienda y Urbanismo, 2008:11-13) son:

1. Municipios: Correspondiente al gobierno local de la comuna. Aplica la Ficha de Protección Social (FPS), entrega información sobre los terrenos y las viviendas, así como los permisos de edificación y avalúos. También recibe las obras y puede prestar servicios de asistencia técnica y social. 
Además de lo anterior, el municipio es responsable de entregar toda la información que le corresponde y sea necesaria para el éxito del proyecto; de otorgar Personalidad Jurídica a los comités; de aplicar y actualizar la Ficha de Protección Social (FPS); de informar la tasación de la vivienda que se quiere comprar; de otorgar el permiso de edificación o construcción; de otorgar la recepción final de las obras, una vez terminada la construcción.

La participación de la Municipalidad en los proyectos habitacionales cumple una función fundamental para la concreción y obtención de la vivienda, y en este sentido puede constituirse como un actor facilitador del proceso o todo lo contrario, puesto que otorga los permisos y requerimientos correspondientes durante las distintas fases del desarrollo del proyecto habitacional.

2. SERVIU: Es el servicio del Estado que depende directamente del MINVU y es responsable de los Programas Habitacionales de la región del país que le corresponde. Revisa los proyectos y solicitudes, paga los servicios de las EGIS y asigna subsidios.

Además de las funciones referentes a permisos y calificación de los proyectos, el SERVIU puede constituirse como un facilitador en el desarrollo de los proyectos, en tanto propicia una colaboración en conjunto con las EGIS.

3. MINVU: El papel de gobierno desde un nivel central a través del MINVU, queda relegado a tres grandes funciones:

- La confección del marco legal que sustenta la política habitacional (en este caso, el decreto 174).

- La coordinación entre los distintos actores.

- La fiscalización para que se cumpla lo planteado por cada una de las partes.

Los principales actores no gubernamentales que establece la política, son:

1. Las Familias Beneficiarias, quienes se organizan en comités de vivienda (con personalidad jurídica) protagonizando el desarrollo del proyecto habitacional. Son quienes deciden, dentro de un cierto marco, dónde estarán localizadas sus viviendas, quiénes se las construirán, qué características tendrán, cómo se configurará el futuro barrio y quién será su Entidad Gestora de Inmobiliaria Social (EGIS).

Lo interesante es observar si las familias beneficiarias son realmente las protagonistas de sus proyectos habitacionales, debido al desbalance de poder propio de la red de actores que conforma la política.

2. Directiva del comité: Son los representantes del grupo organizado de postulantes (Comité), quienes deben recibir y canalizar las inquietudes, consultas y sugerencias de las familias a lo largo de todo el proceso para resolverlas con la EGIS o SERVIU; trabajar permanentemente con la EGIS para el éxito del proyecto; asegurarse de que el proceso se lleve a cabo como está establecido y en los plazos indicados; organizar un sistema de toma de decisiones de la asamblea; administrar con responsabilidad los fondos del Comité.

El rol de la directiva de los comités es clave. No solo por las diferentes gestiones que tienen que realizar para el éxito de los proyectos, sino también por la posición que puedan ocupar en la red de actores. Directivas empoderadas logran apropiarse y hacer suyo el proyecto de vivienda. Lógicamente, el rol que tenga la directiva no solo depende de ellos mismos como comité, sino que también de la lógica de operación de la EGIS que coordina el proyecto.

3. Las EGIS, Pueden ser organizaciones de distinta naturaleza (como ONG's, municipalidades, agrupaciones sociales, Inmobiliarias privadas, etc) que cumplan con los requisitos estipulados por el MINVU y que tengan un convenio firmado con la Secretaría Regional Ministerial (SEREMI) de Vivienda y Urbanismo de la región en la que trabajan; y se ocupan del trabajo técnico y social del proyecto, coordinando a los distintos actores en juego: la constructora, el comité de vivienda, municipio y SERVIUs Regionales en todos los pasos asociados a la obtención de la vivienda definitiva.

4. Empresas Constructoras, los actores provenientes del mercado que por medio de una licitación participan en la construcción de las viviendas.

Las constructoras además de ser las responsables de concretar la construcción de las viviendas, participan en las mesas de trabajo que se realizan de manera trimestral (como mínimo) en conjunto con los demás actores que conforman el proyecto habitacional, siendo parte de las discusiones, negociaciones y toma de decisiones en lo referente a la fase de construcción de las viviendas. 
Además de los actores recién señalados, pueden participar más entidades como otros ministerios, grupos o asociaciones específicas para cada proyecto habitacional en particular.

La fase de implementación o ejecución del programa FSV, se concreta en 4 etapas definidas por el MINVU (2008), -preparación; estudio y calificación; ejecución e; instalación- donde los diferentes actores cumplen un rol más o menos visible según las finalidades en cada una de estas. Sin embargo, son las EGIS y las familias representadas por la directiva del comité, las que protagonizan y tienen mayor participación en las diferentes fases del proyecto habitacional. En este sentido, el tipo de interacciones que se den entre las familias y la EGIS responsable será clave y determinante en la incorporación y vinculación de los demás actores en el proceso.

\section{PRINCIPALES RESULTADOS}

La política habitacional chilena se define y opera en su fase de implementación desde la lógica de coordinación entre los diferentes actores que participan en la red, en concordancia con los requerimientos que surgen de las transformaciones sociales en curso. No obstante, no podemos afirmar desde estos principios de la política habitacional en red, que los procesos deliberativos entre los actores ocurran efectivamente.

Para evidenciar las lógicas de coordinación y organización en red se debe observar, en primer lugar, cómo se concreta y opera la red de actores, porque podría suceder que siga existiendo un único centro rector que controla y administra la coordinación de la red, demostrando una lógica jerárquica en dicho modo de operación.

Bajo este enfoque, es observable de manera paradojal cómo se reproducen lógicas jerárquicas en la instauración de mecanismos formales en el proceso de la política que apelan a la coordinación de actores de tipo no jerárquica, sin embargo, de igual forma son establecidos desde arriba y no desde los actores participantes.

En este contexto, podríamos preguntarnos ¿qué tipo de acciones públicas bajo el enfoque de redes de políticas públicas podrían ser aquellas que potencien mayores niveles de coordinación no jerárquica? En el caso de la política habitacional que aquí se aborda, podríamos pensar en el Plan de Habilitación Social que sustenta esta política, intentando que los proyectos consideren estrategias de superación y promoción de derechos siempre acordes a las características y particularidades del comité de familias que conforma cada proyecto habitacional.

\subsection{Redes de Políticas Públicas en el Programa FSV: Coordinación entre actores y control social.}

En base a las aproximaciones teóricas revisadas, se consideraron los hallazgos que hacen referencia al control social y transparencia en los procesos así como también la propia organización de la red en los proyectos habitacionales.

Como bien se señaló en el marco teórico, la coordinación entre actores es clave en el funcionamiento de la red, reconociendo que existen diferentes formas de coordinación. Ésta puede ser jerárquica y centralizada o desde el otro polo, una coordinación descentralizada y horizontal.

Por tanto, no basta solo con que exista coordinación entre los actores, puesto que la red podría estar liderada por un orden jerárquico en su composición y forma de proceder. Para analizar este punto, debemos tener en cuenta que los actores que participan en la red están definidos desde la normativa que establece la política, pero que la conformación de la misma y el modo de operar de esta, se da según los casos particulares de la misma.

A partir de los resultados obtenidos, podemos establecer que más que una coordinación entre las partes, lo que efectivamente ocurre es un trabajo "compartimentalizado", donde cada uno cumple su función específica sin conversar ni dialogar con los demás actores que conforman la red. Por otra parte, el SERVIU se vincula con los demás actores solo cuando hay conflicto, por lo que no se hacen parte de la red en términos de convivencia ni interacciones posibles. Las familias no tienen un feedback que permita mejorar las políticas, la intervención coordinada de este organismo se limita a los casos conflictivos.

Lo interesante es que desde los casos estudiados en esta investigación, se observa que si bien la relación con el SERVIU no es la más cercana ni la más presente, los distintos actores lo mencionan como un actor clave en el desarrollo de sus proyectos. En varios casos la presencia del SERVIU como miembro de la red ha sido fundamental a lo largo del proceso, e incluso, la mayoría de los proyectos tuvieron un territorial en su momento y al mismo director de la institución visitando su proyecto.

Al parecer, la incertidumbre del SERVIU en tanto el desarrollo de los proyectos habitacionales a través de intermediarios, resulta un aspecto fundamental a considerar. El diagnóstico que ellos realizan sobre la coordinación y la relación entre los actores que participan en la red, no resulta especialmente auspicioso. Faltaría conversación 
entre los participantes, donde cada actor aportase la información que tiene que compartir para estar al tanto de cómo está operando la política, así como también de dar cuenta de posibles problemas o mejoras.

Para que esto ocurra, es necesario crear instancias de comunicación y encuentro que favorezcan una mejor coordinación e interdependencia entre las partes. Para el SERVIU debe ser fundamental que los principales colaboradores sean las familias, pues son ellas quienes tienen la información y puedan estar supervisando en terreno lo que se está haciendo y si se está haciendo como se debería hacer. De ahí la importancia de la participación y de contar con comunidades empoderadas, si tienes una agrupación bien empoderada por lo general tienes buenos resultados.

Desde los casos observados, se confirma la idea de que con dirigentes empoderados los resultados son buenos. Dos de los proyectos que fueron analizados que estuvieron liderados por la EGIS fundación, contaban con una historia de vida en el campamento donde vivían, y ahí la presencia y participación de los dirigentes fue clave. De hecho una de las razones que explicaría el alto nivel participativo en esos proyectos, tiene que ver con el empoderamiento de los dirigentes que a su vez se propagaba en el comité. Así también, uno de los proyectos correspondiente a la EGIS municipal que también tenía estas características, en términos participativos y toma de decisiones a lo largo del proceso, avanzó más allá de los espacios mínimos que el municipio entregaba.

Ahora bien, es posible advertir desde la otra perspectiva, que tener comunidades con dirigentes empoderados podría transformarse en una amenaza para las EGIS y constructoras. Surge así otro elemento interesante de profundizar, puesto que al parecer los intermediarios preferirían comunidades con débil organización comunitaria, que les permitiese tomar sus propias decisiones sin considerar a las familias como las reales protagonistas del proceso.

En este sentido, el trabajo debiera encaminarse hacia el empoderamiento de las familias, que cuenten con la información necesaria y disponible, que sean conscientes de sus deberes y derechos para que puedan administrar su propio capital y miren el proceso sintiéndose dueños del mismo. Sin duda, resultaría fundamental tener buenas EGIS que logren cumplir el Plan de Habilitación Social y trabajar en conjunto con las familias.

\subsection{El control social y transparencia en los procesos}

Cuando una política opera en red y cada actor cumple sus funciones poniendo en juego sus propios intereses y procedimientos, es importante que existan mecanismos de control que permitan transparentar los procesos y recursos que se ponen en juego.

En el caso del programa FSV en general, existen supervisiones de parte del MINVU que tienen por objetivo supervisar que los recursos del Estado se estén utilizando de la forma correcta. Desde los resultados obtenidos de las entrevistas a los funcionarios del MINVU y SERVIU, se observa que en definitiva la relación que se sostiene entre la Institución y los demás actores se concreta en supervisiones de rigor. Para ello existe un supervisor de obras y uno para el área social, quien revisa si el PHS se está o no llevando a cabo de la forma en que se planificó.

Desde el SERVIU se espera que haya un mayor control social para denunciar cuando no están funcionando bien los programas, en tanto los intermediarios e irregularidades en torno al proceso. Ello porque según el diagnóstico realizado por los funcionarios, los resultados de las supervisiones a los intermediarios EGIS señalan que los intermediarios efectivamente no cumplen con lo estipulado, detectando un mal manejo con los recursos en torno a los resultados.

El tema estaría dado por la baja capacidad de control que tienen como Ministerio. Por ende, se esperaría como solución o estrategia no solo dirigentes empoderados, sino también familias que controlen a sus dirigentes.

En este sentido, se vuelve relevante distinguir cuándo un proyecto es exitoso o no en términos de resultados del proceso. Desde los casos estudiados en esta investigación, se da la diferencia. Existen proyectos que se desarrollan en menos tiempo, incluso menos de un año como es el caso de la EGIS privada que se consideró en este trabajo, mientras que existen otros proyectos que tardan más tiempo del estipulado (hasta 3 años algunos), pero que cuentan con procesos participativos más fuertes.

Son muchas las combinaciones que se pueden establecer al respecto. Sin embargo, esta distinción es importante si consideramos los recursos económicos que se asignan para cada proyecto. Es el mismo dinero para un proyecto que dura 9 meses al que dura 2 años o más. Por ende, los recursos para el que dura más son escasos e incluso pueden entrar en una necesidad de suplemento de recursos. Este fue el caso de la EGIS fundación, que si bien destaca por su funcionamiento de la red y por la participación de las familias, han tenido variados problemas 
de recursos en sus proyectos. Al contrario, la EGIS privada que tomó dos casos que arrastraban un fracaso anterior, con los mismos recursos realiza un proyecto habitacional en 9 meses, pero con un bajo nivel de participación de las familias.

\subsection{Organización de la red y coordinación entre actores en los proyectos habitacionales}

En cuanto a la organización de la red y coordinación entre actores, se observan diferencias entre las 3 EGIS.

Los mapas de actores que se presentan a continuación, dan cuenta del tipo de red en cada uno de los proyectos seleccionados. Estos están diferenciados por EGIS y por proyectos.

ESQUEMA N 1

MAPA DE ACTORES PROYECTOS EGIS PÚBLICA

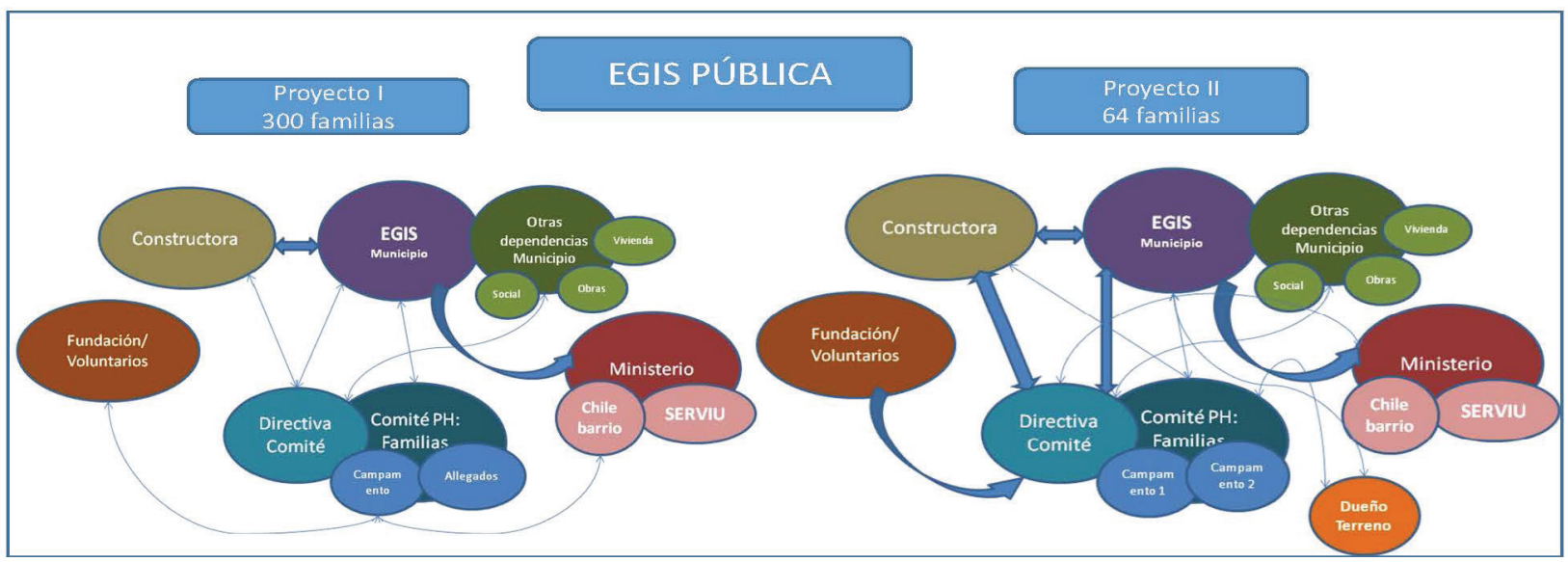

Fuente: Grau, 2013

ESQUEMA N 2

MAPA DE ACTORES PROYECTOS EGIS PRIVADA

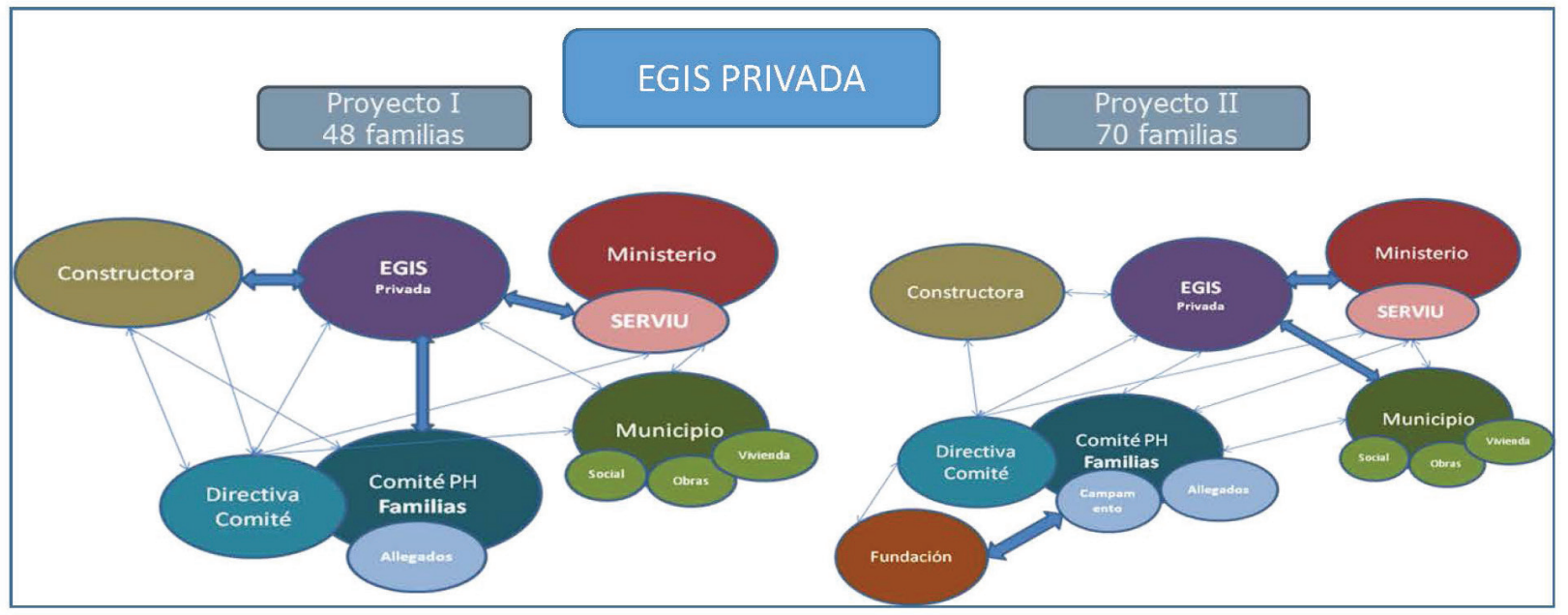

Fuente: Grau, 2013 


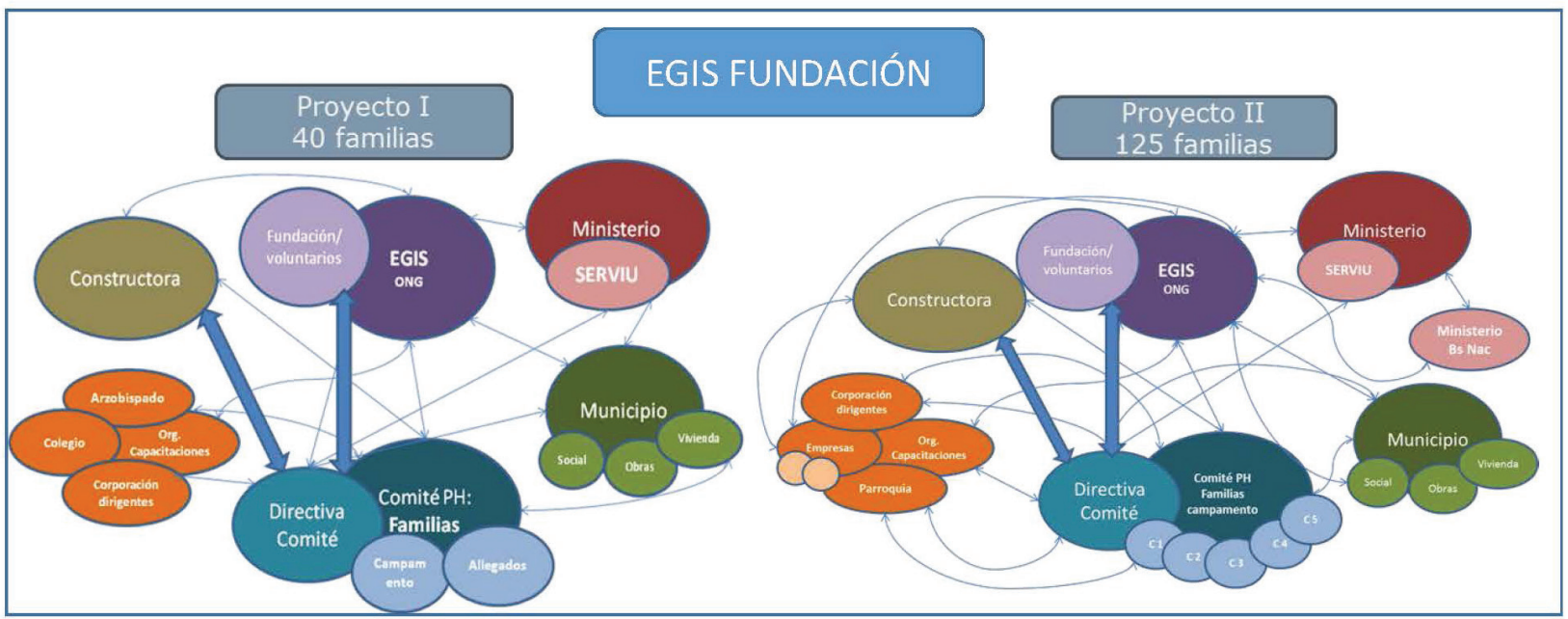

Fuente: Grau, 2013

Se aprecia una tendencia hacia una organización y coordinación en red mayor para la EGIS tipo Fundación, en contraste con lo que se observa en la EGIS privada donde la coordinación entre actores predominaría el ajuste de roles y funciones de cada actor al orden legal vigente. En tanto en la EGIS municipal se observan diferencias entre los dos proyectos abordados. En uno de ellos existiría mayor organización y coordinación, lo que se podría explicar por la organización y tipo de comunidad, con directiva empoderada y partícipe de todo el proceso.

A diferencia de las otras EGIS seleccionadas, en el caso de la EGIS fundación, se destacó en ambos proyectos el número de actores que participaron en la ejecución de estos, mientras que en la EGIS privada solo participaron los definidos según la normativa. Esto podría explicarse a partir de varias razones, por un lado, la fundación no cuenta con recursos económicos suficientes para concluir un proyecto de vivienda que sobrepase los mínimos establecidos en la normativa, lo que requiere de más recursos tanto económicos, humanos y de conocimiento que solo pueden aportar actores privados o públicos. De esta forma, la fundación se asocia a otras entidades que colaboren en el desarrollo de alguna de las áreas del proyecto. Por otro lado, la fundación se sustenta gracias al aporte de terceros, lo que instala una lógica colaborativa que también es parte de los proyectos de vivienda desarrollados por ésta. Lo anterior también se podría visualizar como un aspecto negativo en la medida en que los subsidios entregados no son suficientes para concretar los proyectos habitacionales, de ahí la necesidad de contar con aportes externos para cofinanciarlos.

En ambos casos analizados de la EGIS fundación, se visualiza que no solo la EGIS se vincula con los demás actores participantes, sino también y en forma protagónica, los dirigentes de cada uno de los proyectos. En este sentido, desde las entrevistas realizadas a los dirigentes se destaca la relación con los actores formales e informales que formaron parte del proyecto, instaurando reuniones o espacios de trabajo con cada uno de ellos.

En el caso del proyecto I de esta EGIS, tanto la dirigente como el coordinador social entrevistados señalan la importancia de las interacciones, acuerdos y compromisos atendidos en el proceso. Así también, la relación entre la constructora que ejecutó las obras, con la EGIS y la directiva fue un aspecto vital en el desarrollo del proyecto. La constructora participaba de las mesas de trabajo y asambleas, constituyéndose como un actor más dentro del proceso.

En el proyecto II desarrollado por esta EGIS, la coordinación y colaboración de los distintos actores que participaron en este proyecto, fue fundamental. Una amplia gama de actores externos a los mínimos establecidos por la normativa, aportaron con sus conocimientos y recursos específicos en los distintos momentos del proceso.

En cambio, en el caso de la EGIS municipal, desde las entrevistas realizadas se observa la preponderancia de la jerarquía relativa de cada actor, situando la responsabilidad del proyecto en manos de la EGIS correspondiente que a su vez se vincula fuertemente con el SERVIU como el actor que facilita el proceso. Esto se visualiza aún más desde lo señalado por los coordinadores de los proyectos.

Por otra parte, cabe señalar que en uno de los proyectos de la EGIS fundación existieron fuertes complicaciones que necesitaron de la colaboración de muchos. El comité con la EGIS permitió incorporar variados actores en el proceso, los cuales fueron claves para el logro. 
Las mesas de trabajo y asambleas donde participaban los diferentes actores, se constituyeron en los espacios fundamentales para discutir, dialogar e informar sobre las distintas decisiones y avances del proyecto. Si bien cada actor cumplió su función determinada y aportó en las distintas instancias, la directiva se constituyó como el actor fundamental para el logro del proyecto y clave, para la coordinación entre las partes.

En este sentido, podríamos inferir que la coordinación entre los actores y organización de la red iría más allá de las relaciones regidas desde el orden legal que establece la normativa del FSV, avanzando hacia una coordinación horizontal y descentralizada.

Por otra parte, en el caso de la EGIS municipal, las relaciones entre los actores responden al cumplimiento de lo mínimo establecido por la normativa que regula el funcionamiento del FSV. Esto se concretó en las reuniones estipuladas como justamente necesarias entre los actores participantes. Se observa que predomina la jerarquía relativa de cada actor y que las relaciones se rigen desde el orden legal vigente, desde lo establecido como mínimo, sobre todo al observar la no consideración de las familias beneficiarias en la co-construcción del proyecto.

Otro aspecto relevante en este sentido, tiene que ver con la relación de dependencia que se observa entre las familias y el municipio como institución. Esta relación no cambia ni se modifica en el momento en que la Municipalidad pasa a ser EGIS del proyecto, más bien, se mantiene y se fortalece la lógica del asistencialismo.

Además, desde las entrevistas realizadas, se observa que no existió relación entre las familias y la constructora, ni tampoco se apreció un trabajo ni organización comunitaria entre las familias que conformaban el comité. Todas las actividades e instancias de coordinación se remitieron a lo establecido como mínimo dentro del decreto.

En el caso de esta EGIS existe una diferencia importante entre un proyecto y otro. Esto se podría explicar por el nivel de organización previa que tenían como comunidad.

En el proyecto II correspondiente a la EGIS Municipal, se observa cómo la comunidad convoca y desarrolla distintos espacios de coordinación, negociación y deliberación en conjunto. Si bien este proyecto estaría bajo las mismas condiciones que el anterior, al parecer fue la comunidad quien instauró espacios con la constructora, municipio, alcalde y socios del comité en relación con los avances y decisiones del proyecto. Aunque dista de una coordinación de tipo horizontal y descentralizada, es importante destacar la presencia y participación de las familias en los espacios y decisiones a su alcance. Ello se puede visualizar en la fuerte relación que tenían las familias con la constructora que ejecutó las obras, sin necesitar al municipio como intermediario entre ellos.

Si comparamos la EGIS municipal y fundación con la EGIS privada, observamos que en esta última se distingue un menor nivel organización y coordinación en todas las categorías consideradas en comparación con los demás proyectos correspondientes a la EGIS privada abordados en este trabajo. Sin embargo, ambos proyectos se concretaron con éxito y en los tiempos estipulados una vez que esta EGIS se hace cargo.

Asimismo, en ambos proyectos participaron los mínimos actores establecidos por la normativa, remitiéndose a las labores asociadas de cada rol. Las reuniones que se realizaban entre la EGIS y directiva eran más bien informativas sobre los avances y aspectos claves del proyecto. Sin perjuicio de ello, la directiva destaca en la entrevista la buena relación y disposición de parte de la EGIS para aclarar dudas y realizar consultas respectivas.

Las relaciones entre actores se daban según los requerimientos del proyecto. Por ende, las familias no tuvieron mayor contacto con la constructora a cargo de ambos proyectos, relacionándose esta última directamente con la EGIS y SERVIU. La constructora solo asistió a las reuniones cuando fue necesario.

Sin embargo y a pesar del corto tiempo que duró la ejecución de ambos proyectos con esta EGIS, las familias y dirigentes lograron construir una relación de confianza con la EGIS, no así con la municipalidad.

En el caso del proyecto II, si bien es posible establecer que las familias tuvieron una buena relación con la EGIS, no se visualizaron interacciones positivas con los demás actores, debido a que el comité no confiaba en el municipio por la experiencia anterior de fracaso.

Considerando los puntos recién señalados, se observa la jerarquía relativa de cada actor así como también se observa que la red y sus relaciones se rigen desde el orden legal vigente o de lo establecido como mínimo.

\section{CONCLUSIONES}

El programa FSV se conforma en una red de interacción entre diferentes actores, tanto públicos como privados, que comparten funciones respectivas y responde a su vez, al enfoque de gobernanza democrática. 
La gobernanza en relación a la política habitacional, supone en principio la definición de reglas para la realización de compromisos entre agentes públicos y privados, que permitan una distribución de costos y beneficios frente a una decisión común o una solución de problemas; reglas que en cada caso exigen de los participantes limitar de manera voluntaria su libertad de acción; esto puede conducir a un modelo de identidades organizativas, competencias y esferas de interés mutuamente aceptadas (Mascareño, 2010).

Teniendo en cuenta esta premisa, diremos que no basta con que exista coordinación entre los actores, puesto que la red podría estar liderada por un orden jerárquico en su composición y forma de proceder o por el contrario, una red que opera en forma descentralizada, la cual podría encaminarse a un tipo de coordinación post convencional.

Si bien se apela a una coordinación de actores de tipo no jerárquica para la ejecución de la política, paradójicamente se reproducen lógicas jerárquicas, replicando entonces la lógica de una sociedad administrada - con algunos matices- que genera condiciones ya dadas y no deja espacios para la creación de instancias desde los mismos ciudadanos.

Desde los casos observados, se visualizan distintos tipos de organización de la red según las diferentes EGIS. En el caso de la EGIS fundación, se aprecia mayor descentralización en la coordinación, así como relaciones e interacciones colaborativas y cercanas entre los distintos actores. En tanto, en los proyectos habitacionales correspondientes a la EGIS pública, si bien se observan relaciones cercanas entre comité, EGIS y SERVIU metropolitano, predominó una lógica asistencialista con la municipalidad lo que se transformó en una característica predominante en este caso de estudio, distinguiendo un tipo de normatividad pre convencional con matices convencionales. Los proyectos habitacionales que desarrolló la EGIS privada, constatan que si bien las relaciones con la EGIS eran de confianza, las relaciones e interacciones con el municipio, se configuraban desde la desconfianza.

Sin embargo, estas dimensiones de análisis no solo dependen del tipo EGIS que esté liderando, sino también, de las precondiciones procesuales y normativas de los distintos actores que participan en la red.

Si observamos los resultados de los 6 proyectos habitacionales abordados, se visualizan aspectos comunes en torno a la vida en campamentos y por tanto diferencias entre los comités que no vivieron en estos asentamientos. Así, los proyectos que cuentan con una historia de vida comunitaria, caracterizados por situaciones donde la pobreza moviliza a los ciudadanos, la conformación del comité de vivienda, así como las demás dimensiones analizadas en este estudio, se conforman de una manera distinta.

Para que la política opere desde un enfoque de gobernanza democrática en los aspectos que configuran la red, es fundamental que exista un reconocimiento de la pluralidad de actores, en la asunción de la interdependencia, en la configuración de patrones de conducta que faciliten la participación e interacción de los actores sociales (Kooiman, 1993). Se pone especial atención una crítica o limitación que señalan tanto Mascareño (2010) como Arenilla y Sanchez (2011), sobre la inequidad de los actores participantes en los procesos de operación de una política bajo la lógica de coordinación no-jerárquica, ya que como se puede establecer a partir de los resultados, en el caso específico de la política habitacional chilena y los casos estudiados, los actores sociales no tienen el mismo peso ni influencia en la ejecución de sus proyectos habitacionales y por ende, existiría un claro desequilibrio a favor de los actores públicos o EGIS, lo que reflejaría un referencial normativo de tipo pre convencional o convencional en la red de política. De ahí la relevancia de los intermediarios EGIS en resolver este tipo de inequidades que se dan con frecuencia en una red de política.

Una de las recomendaciones que surgen desde las entrevistas realizadas a los distintos actores, se refiere a la posibilidad de crear instancias de comunicación y encuentro que favorezcan una mejor coordinación e interdependencia entre las partes. En este sentido, la entrevistada del SERVIU sostiene que sería fundamental que los principales colaboradores fueran las familias beneficiarias del programa, lo que implicaría mayores y mejores instancias de trabajo colaborativo y en conjunto entre los diferentes actores, pero dándole un papel protagónico a las familias, representadas por sus dirigentes. De esta forma, la relación con las familias no sería solo en caso de conflicto, sino más bien referente a todo el proceso en que se desarrolla el proyecto habitacional. Lo anterior podría concretarse como un mecanismo formal que supera la incertidumbre sobre el avance y desarrollo de los proyectos y a la vez resguarda el cumplimiento del interés general de la política. 


\section{BIBLIOGRAFÍA}

Abramovich, V. (2006). "Una aproximación al enfoque de derechos en las estrategias y políticas de desarroIlo". Revista de la CEPAL(88), 35-50.

Aguilar, L. (2006). “Capítulo 1 Gobernanza”. En L. Aguilar, Gobernanza y Gestión Pública (págs. 35-136). MexiCO: FCE.

Arellano, J. (2012). Equidad con crecimiento sostenible. Veinte años de políticas sociales Chile 1990-2009. Santiago: Cieplan.

Arenilla, M., \& Sánchez, A. (2011). “El proceso modernizador de la Administración: Andalucía y Castilla y León”. En M. Arenilla, Crisis y reforma de la Administración pública (págs. 139-161). Oleiros: Netbiblo.

Börzel, T. (1997). "Organizing Babylon - On the different conceptions of policy networks". Public Administration, 76(2), 252-273.

Cerrillo, A. (2005). "La Gobernanza Hoy: Introducción”. En A. Cerrillo, La Gobernanza Hoy: 10 textos de referencia (págs. 11-36). Madrid: INAP.

Coleman, J. (1959). "Relational analisis: the study of social organisation with survey methods". Human Organization(17), 28-36.

Dowding, K. (2000). "Modelar metaphor? A critical review of the policy network approach". En P. Dunleavy, P. Kelly, \& M. Moran, British political science: Fifty years of political studies (págs. 196-213). Oxford: Blackwell Publishing.

Grau, M. (2013). Coordinación y Participación en red desde un enfoque de Gobernanza democrática: el caso de la política habitacional chilena 2006 - 2010. Tesis Doctoral. Disponible en: http://eprints.ucm.es/24647/1/T35179. pdf

Jobert, B. (2004). Estado, Sociedad, Políticas Públicas. Santiago: LOM.

Klijn, E. (1998). "Policy Networks: An Overview”. En W. Kickert, y J. Koppenjan, Managing Complex Networks. London: Sage.

Kooiman, J. (1993). Modern Governance: New Government-Society Interactions. Londres: Sage.

Koppenjan, J., Kars, M., \& van der Voort, H. (2009). "Vertical politics in horizontal policy networks: framework setting as coupling arrangement”. Policy Studies Journal, 37(4), 769-792.

Larrañaga, O. (2010). "Las nuevas políticas de protección social en perspectiva histórica”. En O. Larrañaga, y D. Contreras, Las nuevas políticas de protección social (págs. 13-42). Santiago: UQBAR-PNUD.

Mascareño, A. (2010). "Coordinación social mediante políticas públicas: El caso Chileno". Revista de la Cepal(101), 111-125.

Ministerio de Vivienda y Urbanismo. (2008). Manual para Dirigentes y Familias. Fondo Solidario de Vivienda. Santiago: MINVU. CIEPLAN.

Pizarro, C., Raczynski, D., y Vial, J. (1995). Políticas económicas y Sociales en el Chile Democrático. Santiago:

Rhodes, R. (1997). Understanding Governance: Policy Networks, Reflexivity and Accountability. Buckinham: Open University Press-UNPD/PNUD.

Taylor, S., y Bogdan, R. (2002). "La presentación de los hallazgos". En S. Taylor, y R. Bogdan, Introducción a los métodos cualitativos de investigación (págs. 179-185). Barcelona: Paidós.

Weick, K. (1976). "Educational organizations as loosely coupled systems". Administrative Science Quarterly, 21(1), 1-19. 